\title{
Age paternel élevé et risque de schizophrénie
}

\author{
Alexandre Hubert ${ }^{1,2,3,4}$, \\ Andrei Szöke 1,2,3, \\ Marion Leboyer ${ }^{1,2,3}$, \\ Franck Schürhoff ${ }^{1,2,3}$,
}

${ }^{1}$ AP-HP, Groupe Henri Mondor-Albert Chenevier, Pôle de Psychiatrie du CHU de Créteil, Créteil, F-94000, France

2 INSERM Unité 955, IMRB, Département de Génétique, Equipe 15, Créteil, F-94000, France

${ }^{3}$ Université Paris-Est Créteil, Faculté de Médecine, IFR10, Créteil, F-94000, France

${ }^{4}$ Fondation FondaMental, Fondation de coopération scientifique

Correspondance:

Franck Schürhoff, M.D, Ph.D

Pôle de Psychiatrie du CHU de Créteil

Groupe Hospitalier Chenevier-Mondor

40 rue Mesly

94000 CRETEIL, France

Tel: (33 1) 49813051

Fax: (33 1) 49813059

Mail: franck.schurhoff@inserm.fr

Nombre de mots: 4828 


\begin{abstract}
La schizophrénie est une pathologie complexe dont la composante génétique est largement démontrée. Malgré une fertilité réduite, le taux de prévalence reste stable et pourrait s'expliquer par l'apparition de mutations génétiques de novo. Un âge paternel avancé est la source majeure de nouvelles mutations dans l'espèce humaine et pourrait ainsi être associé à un risque accru de développer dans la descendance, une schizophrénie. L’âge de conception allant croissant, il est possible que la population atteinte de schizophrénie augmente également dans les années à venir. L'objectif de ce travail est de faire une revue de la littérature existante sur ce facteur de risque, d'en synthétiser les résultats et de discuter les différentes interprétations avancées. Toutes les études publiées entre 2000 et 2009 ont été sélectionnées via la Bibliothèque Nationale de Médecine (PubMed® base de données). Après avoir contrôlé plusieurs facteurs confondants, il existe un lien significatif ente l'âge paternel et le risque de développer une schizophrénie. Plus l'âge paternel est élevé, plus le risque de développer une schizophrénie est important (11 études sur 13 retrouvent des odd-ratios ou des risques relatifs compris entre 1,84 et 4,62 en faveur d'une augmentation du risque chez les descendants de pères plus âgés au moment de la conception). Compte tenu de ce risque important, plusieurs axes de recherche peuvent être développés : 1) l'identification de manière précise de l'âge à partir duquel le risque est augmenté ; 2) l'identification des phénotypes ou endophénotypes associés à un âge paternel avancé ; 3) les mécanismes qui pourraient expliquer cette augmentation du risque.
\end{abstract}

Mot clés : schizophrénie, âge paternel, mutations de novo, génétique

\title{
Influence of paternal age in schizophrenia
}

Background: Schizophrenia is an aetiologically heterogeneous syndrome, with a strong genetic component. Despite a reduced fertility in this disorder, its prevalence is maintained and could be explained by de novo genetic mutations. Advanced paternal age (APA) is a major source of new mutations in human beings and could thus be associated with an increased risk of developing schizophrenia in offspring. New mutations related to APA have been implicated as a cause of sporadic cases in several autosomal dominant diseases and also in neurodevelopmental diseases, autism, intellectual disabilities, and social functioning. The aim of the present study was to summarize the results of studies investigating the role of APA, and to discuss some interpretations. 
Methods : All relevant studies were identified through the National Library of Medicine (PubMed ${ }^{\circ}$ database). Key-words used for research were "age" and "schizophrenia" linked to "paternal or father". We have identified and analysed 8 cohort studies, 5 case-control studies, 2 meta-analyses, and 1 review concerning different father's mutations potentially transmitted, 2 studies comparing paternal age at conception between sporadic versus familial cases of schizophrenia. All studies selected have been published between 2000 and 2009 .

Results : After controlling for several confounding factors including maternal age, the relative risk of schizophrenia increased from 1.84 to 4.62 in offspring of fathers with an older age of fatherhood. Mother's age showed no significant effects after adjusting for paternal age. There was a significant association between paternal age and risk of developing schizophrenia, there was a weaker association with psychosis.

Discussion : The results of these different studies are confirmed by two recent meta-analyses which found an increased risk of schizophrenia in offspring of father older than 35 years. Two main hypotheses could explain these results. The first one is based on the presence of new mutations in the spermatogonia, possibly because of accumulating replication errors in spermatogonial cell lines. This hypothesis is confirmed by Malaspina et al. (2002), which found that patients without a family history of schizophrenia had significantly older father than probands with a positive family history of schizophrenia. However, this result has not been confirmed by other studies, and paternal age effect could be also explained by a mechanism called imprinting, which is a form of gene regulation. The second hypothesis is based on the fact that father with schizophrenia spectrum personality disorder, known to be genetically related to schizophrenia, could have an advanced age at conception. However, regarding this hypothesis, advanced maternal age at conception should be a risk factor for schizophrenia, and this is not the case. Thus, the first hypothesis seems more plausible than the second one. APA has been identified as a risk factor for other psychiatric disorders such as autism, bipolar disorder, obsessive-compulsive disorder, phobia, and thus seems to be a non specific risk factor. Furthermore, its association with impaired neurocognitive outcomes during infancy and childhood in normal populations raises the question of the phenotype linked to APA.

Conclusion : APA at conception appears to be a risk factor for schizophrenia. This risk factor probably interacts with genetic factors in a gene-environment interaction. To date, there is no validated cut-off at which the risk is significantly increased in offspring. In the future, studies could benefit from analyzing the phenotype related to APA.

Key-words : paternal age, de novo mutations, genetic, schizophrenia. 


\section{I) Introduction}

De nombreuses études ont confirmé l'existence d'une composante génétique à l'étiopathogénie de la schizophrénie (pour revue, [22]). Cette maladie reste complexe et de nombreux gènes sont impliqués sans pour autant qu'un modèle génétique unique puisse expliquer l'ensemble des cas. Malgré tout, l'incidence de cette maladie reste stable dans la population générale, alors que les sujets souffrant de schizophrénie ont moins d'enfants que le reste de la population [8, 23]. Pour expliquer cette situation, certains auteurs ont fait l'hypothèse de l'implication de mutations de novo dont on sait qu'elles augmentent avec l'âge paternel. L’âge paternel avancé est non seulement considéré comme un facteur de risque du développement de la schizophrénie, mais a également été associé à de nombreux troubles neuro-développementaux (diminution des capacités intellectuelles et neuro-cognitives $[1,21$, 30, 31], autisme [30]) ainsi qu'à d'autres pathologies (syndrome d'Apert, Progeria, achondroplasie [12, 36, 40], crâniosynostose [33], situs inversus [16], syndactylie [27], bec de lièvre [26, 32], hydrocéphalie [32], cancer [10, 43]). Une des explications étant la survenue de mutations au niveau des gamètes paternels responsables de l'apparition de cas sporadiques [4].

Parmi les nombreux facteurs de risque mis en cause dans la schizophrénie, Johanson [11] fut le premier à mettre en évidence un lien entre un âge paternel élevé et une augmentation du risque de schizophrénie. Depuis, plusieurs études se sont succédées confirmant ce lien. L'âge paternel à partir duquel le risque de développer une schizophrénie devient significatif varie en fonction des études, mais à l'heure actuelle, aucun âge «seuil»n'a pu être déterminé avec précision. De plus, les hypothèses proposées pour rendre compte de ce lien et leurs implications potentielles pour mieux comprendre la physiopathologie de la schizophrénie ne sont pas univoques.

L'objectif de ce travail est de faire une revue de la littérature existante sur ce facteur de risque, d'en synthétiser les résultats et de discuter les différentes interprétations avancées.

\section{II) Revue de la littérature}

\section{A) Méthode}

Afin d'identifier les articles d'intérêt, nous avons utilisé le moteur de recherche «Medline » en croisant les mots clés suivants: «age » et « schizophrenia » et («paternal » ou «father »). Nous avons pris le parti de sélectionner les articles publiés à partir de 2000 du fait d'une meilleure définition des cas et d'une plus grande rigueur de la méthodologie statistique. Nous avons recensé 15 articles (dont un regroupant 3 études) : 8 études de cohorte, 5 études cas- 
témoins, 2 méta-analyses, 1 revue de la littérature des différentes mutations pouvant être transmises par le père, ainsi que 2 études comparant l'âge du père dans les formes sporadiques et dans les formes familiales de schizophrénie.

\section{B) Présentation générale des études identifiées}

Il existe une grande hétérogénéité des méthodes utilisées pour étudier le lien entre âge paternel et schizophrénie, générant différents problèmes méthodologiques. Par exemple, les critères de définition des cas sont très variables d'une étude à l'autre, certaines études ont intégré des psychoses non-affectives, des psychoses affectives, des troubles schizo-affectifs à leur échantillon. Les différentes critériologies employées et les différents seuils utilisés pour catégoriser l'âge du père à la naissance du sujet varient également. Enfin, le design des études est lui aussi variable : étude cas-témoins, études de cohorte, etc....

Dans la littérature, seules 13 études (analysées au sein de 11 publications) se sont intéressées de manière spécifique au lien entre âge du père et schizophrénie, il s'agit des études de cohorte (au nombre de 8) et des études cas-témoins (au nombre de 5).

a) les études de cohorte

Au total, nous avons identifié 9 articles publiés à partir de l'étude de 8 cohortes différentes (une cohorte ayant fait l'objet de 2 publications [29, 34]). Chronologiquement, la première, celle de Malaspina et al., [18] a porté sur 89.722 sujets nés à Jérusalem et dans ses environs entre 1964 et 1976, et suivis jusqu'en 1997 (the jerusalem perinatal study), ces auteurs identifient 658 individus regroupant des sujets présentant des diagnostics de schizophrénie et des diagnostics apparentés (F20 et F21-F29) de la classification CIM 10. Cette étude montre une corrélation positive entre risque de schizophrénie (et de troubles apparentés) et âge paternel.

Zammit et al., [42] étudiant le devenir de 50.053 militaires suédois engagés entre 1969 et 1970, dont 362 sujets souffrant de schizophrénie et 446 sujets souffrant de psychoses non schizophréniques, mettent en évidence une augmentation significative du risque de schizophrénie et de psychoses non affectives mais dans des proportions plus faibles pour la seconde catégorie, en fonction de l'âge du père (risque multiplié par 3,8 et 1,7 pour les pères de plus de 55 ans par rapport aux pères âgés de moins de 24 ans). Sipsos et al., [34] (étude de cohorte de 754.330 suédois nés entre 1973 et 1980 dont 639 sujets souffrant de schizophrénie et 1311 souffrant de psychoses non affectives) montrent une augmentation significative du risque de schizophrénie (risque multiplié par 4,6 pour les pères de plus de 50 ans par rapport aux pères âgés de 21 à 24 ans). Brown et al., [2], analysent les données concernant 68 sujets souffrant de troubles appartenant au spectre de la schizophrénie (selon le DSM-IV) sur une 
cohorte de 12.094 sujets dont seuls 7709 sont analysés (exclusion des sujets pour lesquels les données maternelles et paternelles sont manquantes et sélection d'un seul sujet par famille). Cette étude retrouve une augmentation du risque de schizophrénie en fonction de l'âge paternel (le risque augmente de 1,8 par tranches de 10 ans ou est multiplié par 3,6 pour les pères âgés de 45 à 68 ans par rapport à ceux âgés de 15 à 24 ans à la conception). Ekeus et al., [6] identifient 366 personnes souffrant de schizophrénie à partir d'une cohorte suédoise de 292.129 sujets nés entre 1973 et 1979 . Ces auteurs identifient un âge maternel et paternel élevés comme facteur de risque de schizophrénie, néanmoins l'influence de l'âge paternel semble supérieure à celle de l'âge maternel. Laursen et al., [15] ont étudié, dans une cohorte danoise de 2.1 millions d'individus nés entre 1955 et 1987, différents facteurs de risque (dont l'âge paternel) pour diverses pathologies psychiatriques (dont la schizophrénie). Cette étude montre une augmentation linéaire du risque de schizophrénie avec l'augmentation de l'âge paternel à la conception (risque multiplié par 1,86 chez les pères âgés de plus de 56 ans par rapport aux pères âgés de 21-25 ans). Enfin, une étude de cohorte américaine de 25.025 sujets nés entre 1959 et 1966, a retrouvé 168 cas de psychoses dont 88 cas de schizophrénie [37]. Ces auteurs retrouvent une tendance, mais ne parviennent pas à mettre en évidence une différence significative entre l'âge paternel des cas et celui des sujets contrôles. El Saadi et al., [7] regroupent trois études dont une étude de cohorte danoise de 2.300.000 individus comprenant 11.672 sujets psychotiques (diagnostic F20-F29, F30-F31, F32.3, F33.3 selon la CIM 10) parmi lesquels 5176 schizophrènes. Cette étude montre une augmentation du risque de psychose lorsque l'âge paternel est $<20$ ans ou $>55$ ans versus un âge paternel situé entre 20-24 ans. Ainsi, ces études de cohorte retrouvent quasiment toutes une augmentation du risque de schizophrénie (ou du spectre de la schizophrénie) avec l'augmentation de l'âge paternel.

b) les études cas-témoins :

Ces études sont au nombre de cinq. Byrne et al., [3] comparent 7.704 sujets souffrant de schizophrénie à 192.590 témoins issus du registre Danois, et montrent une augmentation du risque de schizophrénie, pour un âge paternel supérieur à 50 ans. Dalman et al., [5] étudient 420 sujets souffrant de syndrome schizophrénique (F20, F23.1-23.2, F25 selon la CIM 10), et mettent en évidence un risque de syndrome schizophrénique multiplié par 2,8 chez les descendants de pères de plus de 45 ans par rapport aux pères de 20-24 ans. Enfin, Tsuchiya et al., [38] étudient 99 schizophrènes et 381 témoins : la répartition des âges paternels contrairement aux autres études est faite en 3 groupes ( $\leq 28$ ans, 29-31 ans, $\geq 32$ ans), le risque de schizophrénie est 3 fois plus important pour les pères de plus de 32 ans, comparés 
aux pères de moins 28 ans. El Saadi et al., [7] regroupent trois études dont deux études castémoins: 1) une étude réalisée en Australie comprenant 117 patients (84 schizophrènes, 27 psychoses affectives, 6 sujets psychotiques n'appartenant à aucune des 2 premières catégories, selon les critères du DSM-III-R) ; 2) une étude suédoise de 33.346 individus, dont 539 hospitalisés au moins une fois pour «psychose », et présentant les diagnostics suivants : 154 schizophrènes, 97 troubles bipolaires, 115 autres psychoses affectives, 52 schizophrénies paranoïdes, 103 troubles psychotiques aigus/transitoires, 18 autres psychoses, les autres sujets constituant le groupe témoin. Les auteurs montrent : 1) une absence d'association entre âge paternel et psychose pour la branche australienne; 2) une augmentation du risque de «psychose » lorsque l'âge paternel est $\geq 35$ ans par rapport à un âge paternel de 20-24 ans pour la branche suédoise.

Au total, sur les 13 études issues de 11 articles, plus de la moitié sont des études de cohortes qui ont une méthodologie plus rigoureuse que les études cas-témoins, (absence de biais de sélection dans le recrutement de la population témoin, permettent d'évaluer l'incidence de la maladie sur un groupe exposé et un autre non-exposé, de calculer un risque relatif, sont plus adaptées dans le cadre de maladies fréquentes). On notera également que seules 5 études de cohortes analysent la schizophrénie en tant que telle et non un spectre élargi [6, 15, 34, 37, 42]. Les différentes études vont toutes dans le sens d'une augmentation du risque avec l'augmentation de l'âge du père à la naissance, même si elles utilisent des méthodologies différentes.

\section{C) Synthèses des résultats}

a) Association entre l'âge paternel et le risque de schizophrénie

Plusieurs études dont celle de Malaspina et al., [2, 3, 18] mettent en évidence une augmentation du risque de schizophrénie qui est corrélé positivement avec l'âge du père lors de la conception, et ceci même après ajustement sur l'âge de la mère, le sexe, l'ethnie, le niveau d'éducation, et la durée du mariage des parents. Le risque relatif de schizophrénie est 3 fois plus élevé lorsque le père est âgé de plus de 50 ans par rapport aux pères de moins de 25 ans. Le risque augmente d'un facteur 1,4 par tranche de 10 ans d'âge paternel (après ajustement sur l'âge maternel). Selon, ces auteurs, un âge paternel élevé pourrait être en lien avec $26,6 \%$ des cas de schizophrénies, voire les $2 / 3$ pour les schizophrènes dont les pères ont plus de 50 ans.

Ces données sont confirmées par d'autres études montrant un risque relatif de schizophrénie de l'ordre de 2,4 à 3,8 lorsque le père est âgé de 32 à 55 ans (en fonction des études) au moment de la conception par rapport aux pères de moins 25 ans [2, 3, 5, 7, 34, 38, 42], et ceci 
après ajustement sur l'âge maternel [2, 3, 5, 18, 34, 38, 42]. Plusieurs études montrent également une augmentation modérée du risque chez les pères âgés de moins de 20 ans au moment de la conception [3, 7, 34]. Seules l'étude cas-témoin australienne d'El Saadi et al.,[7] et l'étude de Torrey et al.,[37] ne retrouvent pas d'augmentation du risque en fonction de l'âge du père, probablement du fait d'un manque de puissance statistique.

Afin de clarifier, les différentes données obtenues, nous avons synthétisé l'ensemble des résultats dans le tableau suivant (Tableau I).

\section{INSÉRER TABLEAU I}

L'ensemble des études confirme clairement que l'âge paternel est bien un facteur de risque pour développer une schizophrénie, même si les différents auteurs ne s'accordent pas sur l'importance de ce risque ou sur l'âge à partir duquel ce risque survient.

Un autre point important est que l'influence de l'âge du père paraît surtout plus marquée pour la schizophrénie et semble moindre pour les autres psychoses. Par exemple, il a été montré une augmentation du risque de 1,71 par tranche de 10 ans d'âge paternel dans les psychoses du spectre de la schizophrénie contre 1,89 dans la schizophrénie [2]. De la même manière, Sipsos et al., [34], montrent une augmentation du risque de 1,12 par tranche de 10 ans d'âge paternel pour les psychoses non schizophréniques, non thymiques contre 1,47 pour la schizophrénie. Enfin il n'est pas retrouvé d'augmentation du risque lié à l'âge paternel dans les troubles unipolaires ou bipolaires (à l'exception de la tranche d'âge paternel 51-55 ans pour ces derniers) [15], ni dans les psychoses non schizophréniques [42].

b) Etudes du rôle des facteurs de confusion potentiels dans cette association

\section{Le rôle de l'âge maternel}

La plupart des études s'intéressant au rôle de l'âge paternel comme facteur de risque pour la schizophrénie se sont également intéressées à l'impact de l'âge maternel. Plusieurs études ont montré que l'âge maternel n'est pas associé à une augmentation du risque de schizophrénie [2, 5, 18, 34, 37, 38, 42]. Néanmoins, Byrne et al., [3] mettent en évidence une augmentation du risque de schizophrénie, lorsque la mère a plus de 40 ans, après ajustement sur l'âge paternel et l'existence d'antécédents psychiatriques familiaux, mais cette corrélation disparaît après ajustement sur les facteurs socio-économiques. Une autre étude [6] retrouve une influence de l'âge maternel ( $R R=1,4$ pour les mères de plus de 34 ans), après ajustement sur l'âge paternel, toutefois dans cette étude, l'influence de l'âge de la mère semble moindre en comparaison à celle de l'âge paternel $(\mathrm{RR}=2)$. 


\section{Le rôle du sexe du proposant}

Des résultats contradictoires sont retrouvés concernant l'influence du sexe du sujet atteint [3, 18, 34]. Par exemple, Malaspina et al., [18] mettent en évidence un risque plus élevé chez les garçons (risque multiplié par 1,4 par tranche de 10 ans d'âge paternel contre 1,26 chez les filles), mais ce résultat n'est pas confirmé par d'autres études [34]. Byrne et al., [3] montrent que l'augmentation du risque apparaît pour les filles dont les pères sont âgés de plus de 50 ans et pour les garçons dont les pères sont âgés de plus de 55 ans.

\section{Le rôle des autres facteurs de confusion}

La plupart des études ont évalué le rôle de différents co-facteurs. Par exemple, le risque de schizophrénie lié à l'âge paternel n'est pas modifié par le nombre de frères et sœurs $[2,5,38]$. Seule l'étude de Byrne et al., [3] montre un risque majoré lorsque la fratrie est composée de 3 individus versus un enfant unique, et ceci même après ajustement (sur les antécédents psychiatriques familiaux du $1^{\text {er }}$ degré et les facteurs socio-économiques).

Le statut marital [5] et économique [3, 34], le niveau d'éducation parentale [2, 3], les facteurs démographiques [3], les facteurs saisonniers [3, 34], l'ethnie [2], les facteurs maternonéonataux (les antécédents de psychose maternelle [5], les complications obstétricales [5], le poids de naissance [34], le score d'APGAR [34]), le décès d'un parent avant 18 ans [34] ou avant la première hospitalisation [3], ne modifient pas significativement les résultats concernant l'influence de l'âge paternel sur le risque de schizophrénie.

Certaines études se sont spécifiquement intéressées au rôle des antécédents familiaux, et seront abordées dans la suite de ce travail.

c) Les données issues des méta-analyses

L'ensemble des données issues de la littérature est en faveur d'une influence de l'âge du père pour le risque de développer une schizophrénie. Ces résultats sont corroborés par les études cas-témoins et de cohortes ainsi que plus récemment par deux méta-analyses [37, 41]. La première [41] a été réalisée afin de préciser l'âge paternel à partir duquel il existe une augmentation significative du risque de développer une schizophrénie. Malgré les différences méthodologiques rendant difficile le regroupement des données et leur exploitation, les auteurs retrouvent une majoration du risque de schizophrénie avec l'augmentation de l'âge paternel. Cette augmentation du risque de schizophrénie, particulièrement marqué chez les pères les plus âgés (>54ans), est également présente (de manière plus modérée) chez les pères les plus jeunes ( $<20$ ans). Les auteurs mettent en évidence un risque plus élevé lorsque les pères ont plus de 35 ans, comparés à ceux qui ont moins de 35 ans. Les auteurs concluent qu'un âge paternel supérieur ou égal à 35 ans peut être considéré comme un facteur de risque 
de schizophrénie pour la descendance. Ces résultats sont confirmés par une deuxième métaanalyse qui montre qu'au delà de 35 ans, plus l'âge paternel à la conception est élevé, plus le risque de schizophrénie pour la descendance augmente [37].

\section{III) Discussion et interprétations de résultats.}

Deux hypothèses principales ont été proposées pour rendre compte de ce lien entre âge paternel élevé et schizophrénie. La première hypothèse concerne l'existence de mutations de novo [18]. L'homme est à l'origine de la plupart des nouvelles mutations dans le genre humain. Avant la puberté, les spermatogonies subissent 36 divisions, puis 23 divisions par an. A 20 ans, les cellules germinales masculines ont subi 200 divisions, et 600 à 40 ans. En revanche, chez la femme, les cellules germinales ne subissent que 24 divisions et ceci durant la vie fœtale [4].

Les mutations de novo dont on sait qu'elles augmentent avec l'âge, auraient pour conséquence une influence de l'âge paternel plus importante dans les formes sporadiques par rapport aux formes familiales [18]. Cette hypothèse est corroborée par plusieurs études montrant que l'âge moyen du père dans les formes sporadiques est plus élevé que dans les formes familiales [19], même si ces résultats ne sont pas toujours confirmés [7, 38, 42]. Pulver et al., [28] étudient la réciproque des hypothèses avancées par Malaspina et al., [19] : les apparentés de pères de sujets schizophrènes conçus tardivement devraient avoir un risque familial faible pour la schizophrénie et les troubles apparentés. Ils testent également si l'âge paternel dans les formes familiales est moins élevé que dans les cas sporadiques. Ils ne montrent pas de corrélation entre âge paternel et pathologie schizophrénique chez les apparentés, ni entre âge paternel et antécédents familiaux. Ces résultats ne confirment donc pas ceux obtenus par Malaspina et al., [19] et pourraient être dus à une hétérogénéité clinique des groupes de sujets inclus dans les deux études.

Un effet d'empreinte pourrait également être en cause ici. Il s'agit d'une forme de régulation du génome dans laquelle l'expression du gène dépend du fait que le gène soit hérité du père ou de la mère, lorsque le gène paternel s'exprime le gène maternel est silencieux et inversement, il n'y a donc qu'un allèle parental qui s'exprime, l'autre est rendu silencieux par un processus de méthylation [18]. Les processus de méthylation peuvent être différents en fonction du tissu (cerveau notamment) et du moment du développement. Des « erreurs » lors des ces processus pourraient jouer un rôle dans le développement du langage et du fonctionnement social [35] et donc très vraisemblablement dans la schizophrénie. Il est donc possible qu'interviennent simultanément des mutations de novo et des altérations des 
processus de méthylation [9]. En effet, il existe différents types de DNMTs (DNA methyl transferases), dont le taux et l'activité varient au cours du temps. Des taux et/ou une activité altérés de DNMTs chez les hommes vieillissants pourraient contribuer à une altération des processus épigénétiques en charge d'assurer l'entretien de l'empreinte paternelle durant la spermatogenèse et après la fertilisation [25]. Concernant l'augmentation du risque pour un âge paternel inférieur à 20 ans, il a été proposé comme explication l'immaturité des gamètes, ou une faible activité des enzymes anti-oxydantes ou réparatrices de l'ADN [18].

La deuxième hypothèse concerne l'existence de troubles de la personnalité de type schizoïde ou schizotypique chez les pères des personnes atteintes, ces troubles dont on sait qu'ils appartiennent au spectre génétique de la schizophrénie pourraient expliquer un âge de conception plus tardif. Cependant, cette hypothèse devrait être également associée à un âge maternel élevé comme facteur de risque, or l'âge maternel semble indépendant du risque de survenue de schizophrénie [38, 42].

Une troisième hypothèse peut être évoquée : la perte du père (lié à l'âge de conception avancé) durant le développement psycho-affectif du sujet, ce qui pourrait favoriser des troubles tels que la schizophrénie. Un âge de conception jeune pourrait également être un facteur de risque de séparation des parents et donc une augmentation de risque d'être élevé par une famille monoparentale. La perte d'un parent a été montrée comme étant un facteur de risque pour plusieurs pathologies psychiatriques dont la schizophrénie [15]. Le fait que le décès d'un parent avant 18 ans [34] ou avant la première hospitalisation [3], ne modifie pas l'influence de l'âge paternel sur le risque de schizophrénie, va néanmoins à l'encontre de cette dernière hypothèse.

D'autres hypothèses plus marginales ont également été évoquées. Le délai de conception, lié à des problèmes de fécondité chez l'homme ou chez la femme, pourrait être le facteur de risque, cependant l'effet semble modeste [24]. Un âge paternel avancé ou très jeune est associé à un petit poids de naissance et à une prématurité [17], lesquels pourraient constituer le réel facteur de risque pour les pathologies psychiatriques [13]. La majeure partie de la littérature est en faveur d'une explication génétique ou épigénétique, cette dernière hypothèse permet de faire le lien entre génétique et environnement, et pourrait expliquer les associations retrouvées entre éléments de personnalité prémorbide chez les parents (décrit ci-dessus) et âge paternel avancé. Néanmoins, 1 'épigénétique est difficilement étudiable sur de grand échantillon, et actuellement aucune étude n'a pu démontrer la validité de cette hypothèse.

L'influence de l'âge paternel est mise en cause dans plusieurs maladies psychiatriques comme la schizophrénie, mais également dans l'autisme et plus récemment dans les troubles 
bipolaires [9, 15]. Une étude a montré un lien entre difficultés neuro-cognitives durant l'enfance [31] en population générale et âge paternel avancé : plus ce dernier est élevé, plus le risque de présenter des difficultés neuro-cognitives (par exemple, QI plus faible) est augmenté chez l'enfant (alors que l'âge maternel joue un rôle protecteur). Ces difficultés neurocognitives présentes dans la schizophrénie et l'autisme, pourraient constituer le phénotype associé à l'âge paternel avancé. Une autre étude en population générale portant sur le fonctionnement social chez les adolescents montre un fonctionnement moins bon lorsque leur père, à leur naissance était âgé de moins de 20 ans, ou de plus de 45 ans, et lorsque leur mère était âgée de plus de 40 ans. [39]. Cette étude pose également la question du phénotype associé à l'âge paternel élevé.

Enfin, l'âge paternel avancé comme facteur de risque pose le problème de sa spécificité puisqu'il pourrait être en cause dans les troubles anxio-dépressifs, les phobies et les troubles obsessionnels-compulsifs, avec une augmentation du risque pour ces différentes pathologies lorsque les pères ont moins de 19 ans ou plus de 50 ans [14].

\section{Conclusion}

L'influence de l'âge paternel sur le risque de développer une schizophrénie semble clairement démontrée au vu des différents résultats exposés. L'explication du lien entre âge paternel et schizophrénie reste actuellement au stade des hypothèses, même si une majorité des auteurs semble opter pour une explication génétique. Il est peu probable qu'une seule mutation soit en cause dans les cas de mutations de novo. Le (ou les) gène(s) en cause ne sont actuellement pas connus, plusieurs auteurs soutiennent l'idée d'une interaction gène-environnement. A l'heure actuelle, il n'y a pas de seuil d'âge bien identifiée à partir duquel le risque est important et aucune étude n'a exploré la possible association entre âge paternel avancé et un sous-type clinique particulier de schizophrénie. L'âge moyen de conception des pères allant en augmentant, il serait possible que la prévalence de la schizophrénie augmente dans les décennies à venir [34]. Il serait intéressant que les études futures s'intéressent aux phénotypes associés à un âge paternel avancé, et ce à la fois en terme catégoriel (sous-types selon les classifications internationales) et en terme dimensionnel (prépondérance d'une dimension symptomatique).

Remerciements : Alexandre Hubert a bénéficié pour la réalisation de ce travail d'une bourse de recherche de la Fondation FondaMental ${ }^{\circledR}$ (Fondation de coopération scientifique). 


\section{Références :}

1. Auroux, M.R., et al., Paternal age and mental functions of progeny in man. Hum Reprod, 1989. 4(7): p. 794-7.

2. $\quad$ Brown, A.S., et al., Paternal age and risk of schizophrenia in adult offspring. Am J Psychiatry, 2002. 159(9): p. 1528-33.

3. Byrne, M., et al., Parental age and risk of schizophrenia: a case-control study. Arch Gen Psychiatry, 2003. 60(7): p. 673-8.

4. Crow, J.F., The high spontaneous mutation rate: is it a health risk? Proc Natl Acad Sci U S A, 1997. 94(16): p. 8380-6.

5. Dalman, C. and P. Allebeck, Paternal age and schizophrenia: further support for an association. Am J Psychiatry, 2002. 159(9): p. 1591-2.

6. Ekeus, C., P.O. Olausson, and A. Hjern, Psychiatric morbidity is related to parental age: a national cohort study. Psychol Med, 2006. 36(2): p. 269-76.

7. El-Saadi, O., et al., Paternal and maternal age as risk factors for psychosis: findings from Denmark, Sweden and Australia. Schizophr Res, 2004. 67(2-3): p. 227-36.

8. Fananas, L. and J. Bertranpetit, Reproductive rates in families of schizophrenic patients in a case-control study. Acta Psychiatr Scand, 1995. 91(3): p. 202-4.

9. Frans, E.M., et al., Advancing paternal age and bipolar disorder. Arch Gen Psychiatry, 2008. 65(9): p. 1034-40.

10. Hemminki, K. and P. Kyyronen, Parental age and risk of sporadic and familial cancer in offspring: implications for germ cell mutagenesis. Epidemiology, 1999. 10(6): p. 747-51.

11. Johanson, E., A study of schizophrenia in the male: a psychiatric and social study based on 138 cases with follow up. Acta Psychiatr Neurol Scand Suppl, 1958. 125: p. 1-132.

12. Jones, K.L., et al., Older paternal age and fresh gene mutation: data on additional disorders. J Pediatr, 1975. 86(1): p. 84-8.

13. Kolevzon, A., R. Gross, and A. Reichenberg, Prenatal and perinatal risk factors for autism: a review and integration of findings. Arch Pediatr Adolesc Med, 2007. 161(4): p. 326-33.

14. Krishnaswamy, S., et al., Paternal age and common mental disorders. World J Biol Psychiatry, 2009: p. 1-6.

15. Laursen, T.M., et al., A comparison of selected risk factors for unipolar depressive disorder, bipolar affective disorder, schizoaffective disorder, and schizophrenia from a danish population-based cohort. J Clin Psychiatry, 2007. 68(11): p. 1673-81.

16. Lian, Z.H., M.M. Zack, and J.D. Erickson, Paternal age and the occurrence of birth defects. Am J Hum Genet, 1986. 39(5): p. 648-60.

17. Lundstrom, S., et al., Trajectories leading to autism spectrum disorders are affected by paternal age: findings from two nationally representative twin studies. J Child Psychol Psychiatry, 2010.

18. Malaspina, D., Paternal factors and schizophrenia risk: de novo mutations and imprinting. Schizophr Bull, 2001. 27(3): p. 379-93.

19. Malaspina, D., et al., Paternal age and sporadic schizophrenia: evidence for de novo mutations. Am J Med Genet, 2002. 114(3): p. 299-303.

20. Malaspina, D., et al., Advancing paternal age and the risk of schizophrenia. Arch Gen Psychiatry, 2001. 58(4): p. 361-7. 
21. Malaspina, D., et al., Paternal age and intelligence: implications for age-related genomic changes in male germ cells. Psychiatr Genet, 2005. 15(2): p. 117-25.

22. McClellan, J.M., E. Susser, and M.C. King, Schizophrenia: a common disease caused by multiple rare alleles. Br J Psychiatry, 2007. 190: p. 194-9.

23. McGrath, J.J., et al., The fertility and fecundity of patients with psychoses. Acta Psychiatr Scand, 1999. 99(6): p. 441-6.

24. Opler, M.G., et al., Time-to-pregnancy and risk of schizophrenia. Schizophr Res, 2010. 118(1-3): p. 76-80.

25. Perrin, M.C., A.S. Brown, and D. Malaspina, Aberrant epigenetic regulation could explain the relationship of paternal age to schizophrenia. Schizophr Bull, 2007. 33(6): p. 1270-3.

26. Perry, T.B. and F.C. Fraser, Paternal age and congenital cleft lip and cleft palate. Teratology, 1972. 6(2): p. 241-6.

27. Polednak, A.P., Paternal age in relation to selected birth defects. Hum Biol, 1976. 48(4): p. 727-39.

28. Pulver, A.E., et al., An indirect test of the new mutation hypothesis associating advanced paternal age with the etiology of schizophrenia. Am J Med Genet B Neuropsychiatr Genet, 2004. 124(1): p. 6-9.

29. Rasmussen, F., Paternal age, size at birth, and size in young adulthood - risk factors for schizophrenia European Journal of Endocrinology, 2006. 155(suppl_1): p. 65-69.

30. Reichenberg, A., et al., Advancing paternal age and autism. Arch Gen Psychiatry, 2006. 63(9): p. 1026-32.

31. Saha, S., et al., Advanced Paternal Age Is Associated with Impaired Neurocognitive Outcomes during Infancy and Childhood. PLoS Med, 2009. 6(3): p. e40.

32. Savitz, D.A., P.J. Schwingl, and M.A. Keels, Influence of paternal age, smoking, and alcohol consumption on congenital anomalies. Teratology, 1991. 44(4): p. 429-40.

33. Singer, S., et al., Craniosynostosis in Western Australia, 1980-1994: a populationbased study. Am J Med Genet, 1999. 83(5): p. 382-7.

34. Sipos, A., et al., Paternal age and schizophrenia: a population based cohort study. Bmj, 2004. 329(7474): p. 1070.

35. Skuse, D.H., et al., Evidence from Turner's syndrome of an imprinted X-linked locus affecting cognitive function. Nature, 1997. 387(6634): p. 705-8.

36. Tolarova, M.M., et al., Birth prevalence, mutation rate, sex ratio, parents' age, and ethnicity in Apert syndrome. Am J Med Genet, 1997. 72(4): p. 394-8.

37. Torrey, E.F., et al., Paternal age as a risk factor for schizophrenia: how important is it? Schizophr Res, 2009. 114(1-3): p. 1-5.

38. Tsuchiya, K.J., et al., Advanced paternal age associated with an elevated risk for schizophrenia in offspring in a Japanese population. Schizophr Res, 2005. 76(2-3): p. 337-42.

39. Weiser, M., et al., Advanced parental age at birth is associated with poorer social functioning in adolescent males: shedding light on a core symptom of schizophrenia and autism. Schizophr Bull, 2008. 34(6): p. 1042-6.

40. Wilkin, D.J., et al., Mutations in fibroblast growth-factor receptor 3 in sporadic cases of achondroplasia occur exclusively on the paternally derived chromosome. Am J Hum Genet, 1998. 63(3): p. 711-6.

41. Wohl, M. and P. Gorwood, Paternal ages below or above 35 years old are associated with a different risk of schizophrenia in the offspring. Eur Psychiatry, 2007. 22(1): p. 22-6.

42. Zammit, S., et al., Paternal age and risk for schizophrenia. Br J Psychiatry, 2003. 183: p. 405-8. 
43. Zhang, Y., et al., Parental age at child's birth and son's risk of prostate cancer. The Framingham Study. Am J Epidemiol, 1999. 150(11): p. 1208-12. 
Tableau I : Risque (Odds-ratios (OR) ou Risque relatif(RR)) de schizophrénie en fonction de l'âge paternel.

\begin{tabular}{|c|c|c|c|c|c|c|}
\hline Etudes & Lieu & $\begin{array}{c}\text { Type } \\
\text { d'étude }\end{array}$ & $\begin{array}{c}\text { Population } \\
\text { d'intérêt } \\
\text { (nombre de cas) }\end{array}$ & $\begin{array}{l}\text { Nombre de } \\
\text { sujets }\end{array}$ & OR/RR (IC 95\%) & Diagnostic \\
\hline $\begin{array}{l}\text { El Saadi (2003) (étude } \\
\text { 1)[7] }\end{array}$ & Danemark & \multirow{8}{*}{ Cohorte } & 11.672 & 2.300 .000 & $1,84(1,33-2,53)^{4}$ & F20-F29, F30-F31, F32.3, F33.3 ${ }^{12}$ \\
\hline Sipsos (2004)[34] & Suède & & 639 & 754.330 & $4,62(2,28-9,36)^{6}$ & $\mathrm{~F} 20^{12}$ \\
\hline Malaspina (2001)[20] & Israël & & 658 & 87.906 & $2,96(1,60-5,47)^{4}$ & F20 ; F21-F29 ${ }^{12}$ \\
\hline Zammit (2003)[42] & Suède & & 362 & 50.053 & $3,8(1,83-11,8)^{1}$ & $\mathrm{~F} 20^{12}$ \\
\hline Brown (2002)[2] & $\begin{array}{l}\text { Etats- } \\
\text { Unis }\end{array}$ & & 68 & 7.641 & $3,6(0,4-33,9)^{2}$ & $295-299^{11}$ \\
\hline Ekeus (2006)[6] & Suède & & 366 & 292.129 & $1,9(0,5-7,8)^{7}$ & $\mathrm{~F} 20^{12}$ \\
\hline Torrey (2009)[37] & $\begin{array}{l}\text { Etats- } \\
\text { Unis }\end{array}$ & & 88 & 25.025 & $1,06(0,59-1,94)^{10}$ & $\mathrm{~F} 20, \mathrm{~F} 25.1^{12}$ \\
\hline Laursen (2007)[15] & Danemark & & 13.297 & $>2.100 .000$ & $1,86(1,39-2,50)^{3}$ & $\mathrm{~F} 20^{12}$ \\
\hline Byrne (2003)[3] & Danemark & \multirow{5}{*}{ Cas témoins } & 7.704 & 192.590 & $2,42(1,56-3,77)^{4}$ & $\mathrm{~F} 20^{12}$ \\
\hline Dalman (2002)[5] & Suède & & 420 & 857 & $2,8(1,3-6,3)^{8}$ & $295-298^{11}$ \\
\hline $\begin{array}{l}\text { El Saadi (2003) (étude } \\
\text { 2)[7] }\end{array}$ & Suède & & 134 & 8.678 & $2,42(1,19-4,89)^{5}$ & $\begin{array}{l}295-299^{11} \\
\text { F20 }\end{array}$ \\
\hline $\begin{array}{l}\text { El Saadi (2003) (étude } \\
\text { 3)[7] }\end{array}$ & Australie & & 117 & 146 & $0,77(0,24-2,46)^{5}$ & $\begin{array}{r}\text { F20, F23.2, F22.0, F25, F29, F30.2, F31.2, F31.5, F32.3, } \\
\text { F33.3 }\end{array}$ \\
\hline Tsuchiya (2005)[38] & Japon & & 99 & 381 & $3(1,49-6,04)^{9}$ & $295^{11}$ \\
\hline
\end{tabular}

${ }^{1}$ âge paternel $\geq 55$ ans vs $15-24$ ans 2 âge paternel $\geq 45-68$ ans vs $15-24$ ans

3 âge paternel $\geq 56$ ans vs $21-25$ ans

${ }^{4}$ âge paternel $\geq 55$ ans vs $20-24$ ans

5 âge paternel $>35$ ans vs $20-24$ ans

${ }^{6}$ âge paternel $\geq 50$ ans vs $21-24$ ans

${ }^{7}$ âge paternel $\geq 50-54$ ans vs 25-29 ans

8 âge paternel $\geq 45$ ans vs $20-24$ ans

\section{${ }^{11}$ CIM9}

295 : Psychoses schizophréniques

296 : Psychoses affectives

297 : États délirants

298: Autres psychoses non organiques

299 : Psychoses spécifiques de l'enfance

\section{${ }^{12} \mathrm{CIM} 10$}

F20-29 : Schizophrénie, troubles schizotypiques et troubles délirants

F20 : Schizophrénie

F21 : Trouble schizotypique

F22.0 : Trouble délirant

F23: Troubles psychotiques aigus et transitoires

F23.2 : Trouble psychotique aigu d'allure schizophrénique

F25 : Troubles schizo-affectifs 
${ }^{9}$ âge paternel $\geq 32$ ans vs $\leq 28$ ans

âge paternel $\geq 35$ ans vs $\leq 35$ ans
F25.1 : Trouble schizo-affectif, type dépressif

F29: Psychose non organique, sans précision

F30 : Épisode maniaque

F30.2 : Manie avec symptômes psychotiques

F31: Trouble affectif bipolaire

F31.2 : Trouble affectif bipolaire, épisode actuel maniaque avec symptômes psychotiques

F31.5 : Trouble affectif bipolaire, épisode actuel de

dépression sévère avec symptômes psychotiques

F32.3 : Episode dépressif sévère avec symptômes

psychotiques

F33.3 : Trouble dépressif récurrent, épisode actuel sévère

avec symptômes psychotiques 